\title{
Relative Disparity Processing in the Dorsal Visual Pathway
}

\author{
Matthew L. Patten ${ }^{1}$ and Aidan P. Murphy ${ }^{1,2}$ \\ ${ }^{1}$ School of Psychology, University of Birmingham, Edgbaston, Birmingham, B15 2TT, United Kingdom, and ${ }^{2}$ Section on Cognitive Neurophysiology and \\ Imaging, Laboratory of Neuropsychology, National Institute of Mental Health, Bethesda, Maryland 20892 \\ Review of Krug and Parker
}

Binocular disparity results from the subtle differences between the two eyes' views, and provides a powerful cue to the depth structure of the world around us. Disparity is widely conceptualized as having two types of signal: absolute disparity, which corresponds to the retinal distance of objects from an observer's current point of fixation, and relative disparity, which corresponds to distances between objects independent of where the eyes are fixating. The calculation of relative disparity therefore relies on absolute disparity information. The processing of these two signals has been suggested to differ between two visual pathways, with absolute disparity processed predominantly in the dorsal stream for visually guided actions (e.g., navigation, grasping, vergence eye movements) and relative disparity processed in the ventral stream for the identification of objects (e.g., 3D shape processing, object recognition) (Neri et al., 2004; Anzai et al., 2011). Previous studies investigating relative disparity computations have used a center-surround stimulus configuration, assuming this reflects receptive field organization (Uka and DeAngelis,

Received Feb. 7, 2012; revised March 5, 2012; accepted March 6, 2012.

This work was supported by the European Community's Seventh Framework Programme (FP7/2007-2013, Grant Agreement No. 214728-2, to M.L.P.) and a Wellcome Trust-NIH studentship (WT091467MA, to A.P.M.). We thank Andrew Welchman for helpful comments.

The authors declare no competing financial interests.

Correspondence should be addressed to Matthew L. Patten, School of

Psychology, University of Birmingham, Edgbaston, Birmingham, B15 2TT, United Kingdom. E-mail: m.l.patten@bham.ac.uk.

DOI:10.1523/JNEUROSCI.0588-12.2012

Copyright $\odot 2012$ the authors $\quad 0270-6474 / 12 / 325353-03 \$ 15.00 / 0$
2006; Anzai et al., 2011). However, a failure to detect relative disparity processing in dorsal visual areas by presenting a center-surround stimulus might reflect suboptimal neuronal stimulation rather than different types of disparity computation.

In a recent issue of The Journal of Neuroscience, Krug and Parker (2011) developed a novel stimulus configuration to test whether there is selectivity for relative disparity in area V5/MT, which is located in the dorsal visual pathway. They recorded individual neurons in awake, fixating macaque monkeys when two superimposed transparent planes were presented. These planes had different depths and contained dots moving in opposite directions. The stimulus set typically consisted of eight absolute disparities with varying relative depths. A disparitytuning curve was generated by plotting firing rate against absolute disparity. Disparities were then added to the entire stimulus set to change its position in depth. For a relative-disparity-selective neuron, this manipulation shifts the position of the tuning curve, because the neuron responds to the depth between the two planes. In contrast, the tuning curve of a neuron selective for absolute disparity does not shift when at a new depth position, because such neurons' absolute disparity preferences remain the same. The shift in tuning curves was quantified with a metric used by previous studies to allow comparison of results (Krug and Parker, 2011, their Fig. 9). Overall, the authors found a significant shift in tuning curves for the population of V5/MT neurons tested, indicating selectivity for relative disparity in this area. However, the amount of selectivity to relative disparity varied between neurons, with some being tuned more closely to absolute disparity.

In contrast to these results, previous studies that directly tested relative disparity selectivity in neurons of dorsal visual areas reported no such selectivity (Uka and DeAngelis, 2006; Anzai et al., 2011). Uka and DeAngelis (2006) used a more conventional center-surround stimulus configuration to measure neuronal responses to absolute and relative disparity in V5/MT. Krug and Parker (2011) attribute the observed difference in results to this difference in stimulus configuration. However, it is not clear why this would alter the relative disparity selectivity of V5/MT neurons, rather than produce a net difference in spike rates. Further, several functional imaging studies in which non-center-surround stimuli were presented have also reported a lack of sensitivity to relative disparity at a population-encoding level in human V5/hMT+ (Backus et al., 2001; Neri et al., 2004), although it is possible this could represent a difference in processing between macaque and human V5/hMT+.

A unique aspect of the experiment by Krug and Parker (2011) was the use of two superimposed planes. In a second experiment, the authors measured the neuronal responses to each of the planes individually and, by testing different linear combinations, they attempted to reproduce the 
response of the two-plane stimulus. The presence of a second plane-located at a different depth and moving in the opposite direction-is likely to change a neuron's response from that obtained when a single plane is presented. In particular, the presence of both planes should alter the response of a neuron that is selective to relative disparity. Krug and Parker (2011) found a better prediction for the neuron's response if both planes were taken into account and even more if an interaction term was included (although this amount varied between neurons). The authors concluded that the neuron's responses could not be adequately explained by a linear combination of its constituents. The inclusion of an interaction term significantly increased the fraction of response variance accounted for (from a median value of 0.65 to 0.73 ). However, it would have been appropriate to have used an information criterion to determine whether the improvement observed justifies the increased dimensionality from an additional parameter in their regression model.

The authors also found that the inclusion of a second plane did not generally change the disparity preference of the neuron, although it altered the steepness of the tuning curve. Importantly, this change in steepness of the tuning curve was correlated with relative disparity selectivity. This result is particularly striking in suggesting that relative-disparitysensitive V5/MT neurons modify their response depending on the type of disparity signal present. However, we cannot assume this change directly relates to the processing of relative disparity. The presence of both planes also introduces motion opponency in the stimulus, which has been observed to affect neuronal responses in V5/MT when no disparity was presented (Recanzone et al., 1997).

The two-plane stimulus used by Krug and Parker (2011) shares similarities in configuration to a transparent rotating cylinder stimulus: namely, both stimuli include transparent motion in opposite directions at different depths. The cylinder stimulus additionally contains structurefrom-motion, a depth cue that V5/MT neurons are known to encode (Dodd et al., 2001). When disparity is removed from this stimulus, the front and rear surfaces are rendered ambiguous and the direction of rotation becomes perceptually bistable. Relative disparity selectivity in V5/MT neurons might reflect the processing of depth order in general, regardless of which depth cue (disparity or structure- from-motion) is available. To test this, Krug and Parker (2011) recorded responses from a subset of V5/MT neurons while monkeys made perceptual judgments of ambiguous rotating cylinder stimuli. This suggested that V5/MT neurons encoded the perceived direction of rotation for the cylinder, although no correlation with relative disparity selectivity was found. This indicates that both absolute- and relative-disparity-tuned V5/MT neurons contribute to this task. However, this was tested in the absence of a disambiguating cue (disparity), and therefore does not necessarily reveal how V5/MT cells that are selective for different disparity types might integrate depth from motion and disparity signals.

One possibility is that the two-plane stimulus used by Krug and Parker (2011) engages neurons involved in the integration of binocular disparity and motion parallax depth cues. Recent human fMRI data indicate the involvement of dorsal regions $(\mathrm{V} 3 \mathrm{~B} / \mathrm{KO})$ in this process, although the human homolog V5/hMT+ appears to play a less crucial role (Ban et al., 2012). However, V5/MT neurons encode motion parallax and structure-from-motion signals when no disparity is present (Dodd et al., 2001; Nadler et al., 2009) and might therefore be expected to change their response to a disparity stimulus when a motion signal consistent with depth ordering is added. Testing for relative disparity selectivity using stimuli composed of transparent planes moving in the same, rather than opposite, directions will help to determine whether this configuration elicits responses related to motion parallax.

The results of Krug and Parker (2011) justify a revised view of the cortical organization of disparity processing. Specifically, the results indicate that relative disparity can be processed in the dorsal pathway. Converging lines of evidence support this conclusion. In a recent study, Cottereau and colleagues (2011) took a novel approach by measuring the temporal dynamics of disparity processing using EEG. They compared responses to a center-surround stimulus when the surround was composed of uncorrelated noise (absolute disparity) versus a correlated surround (relative disparity). They found that the change in stimulus altered the temporal characteristics of the signal from the region of interest, V5/hMT + , introducing a lag of $\sim 30 \mathrm{~ms}$, which they attributed to the calculation of relative disparity.

Further evidence of sensitivity to relative disparity in dorsal regions comes from studies in which cortical responses to stereoscopically defined 3D shapes presented at different positions in depth were measured. Processing the 3D structure of such objects regardless of their distance from fixation would require information about the relative disparity of adjacent surface points, including disparity gradients. The results indicated that $3 \mathrm{D}$ structure from relative disparity is encoded in parietal regions (Srivastava et al., 2009) as well as V5/MT (Nguyenkim and DeAngelis, 2003), although the same parietal regions also encode $3 \mathrm{D}$ structure specified by other depth cues.

Using a novel stimulus configuration, Krug and Parker (2011) observed that a large proportion of neurons in the dorsal stream can exhibit selectivity for relative disparity. In line with traditional characterization of cortical visual processing pathways, they propose that relative disparity is likely to serve different functions between the visual streams: for navigation and segmentation of moving objects in the dorsal stream and for 3D shape perception in the ventral stream. The discrepancy between these results and those of a previous study likely resulted from differences in stimulus configuration and demonstrates the complexity of processing that occurs in the extrastriate visual cortex. Future research should systematically examine the effect particular stimulus properties have on the neuronal responses to relative disparity in V5/MT and other visual areas.

\section{References}

Anzai A, Chowdhury SA, DeAngelis GC (2011) Coding of stereoscopic depth information in visual areas V3 and V3A. J Neurosci 31:10270-10282.

Backus BT, Fleet DJ, Parker AJ, Heeger DJ (2001) Human cortical activity correlates with stereoscopic depth perception. J Neurophysiol 86:2054-2068.

Ban H, Preston TJ, Meeson A, Welchman AE (2012) The integration of motion and disparity cues to depth in dorsal visual cortex. Nat Neurosci 15:636-643.

Cottereau BR, McKee SP, Ales JM, Norcia AM (2011) Disparity-tuned population responses from human visual cortex. J Neurosci 31:954-965

Dodd JV, Krug K, Cumming BG, Parker AJ (2001) Perceptually bistable three-dimensional figures evoke high choice probabilities in cortical area MT. J Neurosci 21:4809-4821.

Krug K, Parker AJ (2011) Neurons in dorsal visual area V5/MT signal relative disparity. J Neurosci 31:17892-17904.

Nadler JW, Nawrot M, Angelaki DE, DeAngelis GC (2009) MT neurons combine visual motion with a smooth eye movement signal to 
code depth-sign from motion parallax. Neuron 63:523-532.

Neri P, Bridge H, Heeger DJ (2004) Stereoscopic processing of absolute and relative disparity in human visual cortex. J Neurophysiol 92:1880-1891.

Nguyenkim JD, DeAngelis GC (2003) Disparitybased coding of three-dimensional surface orientation by macaque middle temporal neurons. J Neurosci 23:7117-7128.

Recanzone GH, Wurtz RH, Schwarz U (1997) Responses of MT and MST neurons to one and two moving objects in the receptive field. J Neurophysiol 78:2904-2915.

Srivastava $S$, Orban GA, De Mazière PA, Janssen P (2009) A distinct representation of three- dimensional shape in macaque anterior intraparietal area: fast, metric, and coarse. J Neurosci 29:10613-10626.

Uka T, DeAngelis GC (2006) Linking neural representation to function in stereoscopic depth perception: roles of the middle temporal area in coarse versus fine disparity discrimination. J Neurosci 26:6791-6802. 